\title{
Foundations for Science of Information: Reflection on the Method of Inquiry
}

\author{
Marcin J. Schroeder \\ mjs@aiu.ac.jp, Akita International University, Akita, Japan
}

\begin{abstract}
The paper considers necessary conditions for establishing information science as a scientific autonomous discipline. The lack of a commonly accepted definition of information is not as threatening as it may seem, as each study within the discipline may choose an own definition, as well as an own philosophical framework, when there are some alternatives to choose between. More important is the development of a common methodology of inquiry and some range of standard questions regarding the concept of information. Also, it is important to develop some standards of inquiry, which would make information scientific studies accessible to philosophical analysis and reflection. In turn, contributions of information science to the resolution of problems identified within philosophy will give the best measure of maturity for information science as a discipline.
\end{abstract}

Keywords: Methodology of Information Science, Philosophy of Information Science, Definition of Information

Information does not have yet a commonly accepted, generic, context independent definition. This is not as disturbing as it may seem for the emancipation of information science. After all, empirical disciplines do not start from precisely formulated definitions, but from the evidence of reoccurring patterns of phenomena and attempts to build appropriate conceptual framework to analyze them.

What actually is necessary for a rising discipline of information science in order to secure its identity are a widely accepted range of instances of the occurrence of information or information phenomena and a common agreement regarding the earlier results of inquiry, usually carried out within other fields, that should be either adopted into the new discipline, or at least explained by it. For instance, it is difficult to imagine science of information disregarding completely Shannon's entropy as a measure of information, although further developments may replace it by a more suitable measure.

Thus, even if the temperature of the discussions on the definition of information is continuously high, there is little disagreement regarding what phenomena should be considered as information related, and which results qualified as studies of information have lost relevance for the future discipline.

Accepted as a matter of fact, current lack of commonly accepted definition of information is by no means a justification for disregard of the importance of the search of such a definition, or possibly definitions which could direct more domain specific studies of information. Even more important is that no study should avoid clear statement of its way of understanding of this fundamental concept or resort to casual, common sense, ill defined, but catchy explanation of the meaning.

In this paper, there will be frequent references to author's own definition (Schroeder, 2005) introduced in the past, but many theses discussed here are independent from the specifics of this definition. The references to this definition and to the philosophical foundations on which it has been built serve rather as exemplifications of what is presented in more general manner allowing for different understanding of information.

Philosophical foundations for the study of information are mentioned above not accidentally. Together with the choice of a particular definition of information, in every information-scientific study it should be clear what conceptual framework is used, and this requires that some philosophical foundations are established. Too frequently, papers about information addressing fundamental issues, in apparent attempt to preserve generality, avoid specification of the meaning of fundamental concepts, which at best is the result of reluctance to declare own position to avoid controversy, 
at worst of intellectual poverty. In the consequence, frequently in one paper there are references to the results coming from works built on contradictory assumptions threatening consistency of the reasoning.

Although it is not necessary (not even desirable or possible) for scientific discipline, in distinction from its particular studies, to be funded on a unique philosophical platform, it has to have a definite methodology (including a register of basic questions) and its theses should allow for philosophical interpretation and reflection. Its maturity can be measured by the number and extent of its contributions to philosophical inquires, especially those motivated by questions of independent origins.

\section{Scientific Methods of Information Studies}

The explosion of interest in information started from Shannon's epoch making paper on his Theory of Communication. It is quite clear that he was writing about communication and that his work was not a theory of information, although he is using this expression when introducing "[q]uantities of the form $\mathrm{H}=-\Sigma \mathrm{p}_{\mathrm{i}} \log \mathrm{p}_{\mathrm{i}}[\ldots]$ as measures of information, choice and uncertainty" (Shannon, 1949/1998). The word "information" appears there in the compound of "information source which produces a message" and it is information source which is modeled mathematically as a Markoff process, not information. But the fact that something is measured, be it information, chance or uncertainty, gave existence to the new concept and at the same time new entity, something which exists independently and which has a quantitative characteristic called entropy. It is not a message, as in the process of communication it is changed into a signal from which it is later reconstructed as a message again. It would be strange to consider choice or uncertainty moving or being transformed, so without clear statement in Shannon's work this entity has been identified with information. Shannon's entropy acquired dozens of generalizations, but remains a fundamental, although not unquestionable measure of information. Present author's view is that it does not measure information, but the capacity of a carrier to acquire information (Schroeder, 2004).

For several decades the study of information has been dominated by the exploitation of the quantitative characteristics of information, including attempts to make it independent from the probability measure which has to be pre-defined for the purpose of introducing of the concept of entropy or its generalizations. The attempts to study of information as a structural concept have acquired relatively little attention, at least among those interested in information as a fundamental idea, due to open disregard for immense volume of results based on the quantitative approach.

However, the actual theory of information must provide not only quantitative description, but also model or models of the concept itself. It is not enough to say that entropy measures information, if we do not have any mathematical (or other) representation of the concept itself. Can we say that a probability measure is information? Entropy is just a single numerical characteristic of a probability measure. If we do not agree that probability measure is information, then what it is?

Here we have the point where the need for methodological reflection becomes clear. What are the characteristics of information different from its measure? What do we want to know about information?

We have to start from the recognition of the possibility that there are some other quantitative descriptions of information. Kolmogorov-Chaitin algorithmic measure is an example, although not perfect, as we can find its close correspondence to the original one. Both are measuring size of information. We may have quantitative measures which are not related to the size, such as for instance ill-fated measure of semantic information developed by Bar-Hillel and Carnap (1952/1963). Development of other quantitative characteristics requires prior answers to the questions what do we want to measure and how? But this question should be considered only after we can clarify more general issue of characteristics which may have only qualitative expression.

Thus, what kind of questions can we ask about information? What are the main features of this concept? We can try to use experience of another discipline, but closely related, semiotics. It is natural to inquire about structural characteristics of information analogical to those considered in syntactics of a symbolic system, i.e. the ways information is build of some structural units. Another 
perspective can be given by consideration of localization, and corresponding to it distribution, of information, both referring to either the spatial (geometric, topological, or other,) or temporal description. Yet another approach would be an analogue to formal logical analysis of information. In this case it would be necessary to develop a concept of informational consequence.

Different direction, based on the analysis of the meaning of information, has been already initiated right after the publication of Shannon's paper. Very early attempts of Bar-Hillel and Carnap belong to this direction, but the lack of convincing results inhibited the development of semantic analysis. Success of such an approach requires an answer to the question about the informationscientific understanding of symbolic representation which is in the core of the problem. The present author has proposed an approach based on a new concept of the symbol as a representation of big volume of information on one carrier by a smaller one on a different carrier (Schroeder, 2010).

Pragmatics of information is probably at present the most active and developed direction of study, at least when it is understood in terms of the use of information in human collectives, or alternatively in terms of its use in computer technology. The approach could be generalized to the question of functioning of information in the external context of information and informational processes.

The attempts to answer the questions about characteristics of information should start from some analysis of the manifestations of information. Shannon's measure of information has been based on the choice of manifestation which can be called selective. Information source is selecting (making choices in Shannon's words) of specific options out of predetermined collective according to some probability distribution. This dependence on a probability distribution has been a source of opposition among those who were more interested in information which can be called structural, and which is reflected in the mutual relationship of the elements of some collective (Schroeder, 2005). Kolmogorov-Chaitin concept of algorithmic information investigates structural characteristics of the code, but is limited to very limited type of structural relationships which do not deviate from the original concept of a message. Structural characteristics may go much further in the direction of geometric, topological, or even more general relations.

Looking at the way information has been exploited by other civilizations, we may think about a type of information manifestation which could be called "taxonomic". Majority of cultures have accumulated experience by grouping particular instances of information around representative prototypes. Such "ethnoscientific" information systems studied mainly with the use of linguistic tools, sometimes acquired level of very high sophistication exceeding that of the western style scientific disciplines. Frake (1962) gives example of Hanoonoo tropical-forest agriculturalists of the central Philippines who classify their plant world into more than 1600 categories, whereas systematic botanists classify the same flora into less than 1200 species. There is of course a question whether this information structure is a reflection of the characteristics of information inherent in the environment or rather human, cultural invention resulting from the necessity to deal with the rich and unorganized volume of information overflowing genetically transmitted mechanisms of information processing.

There is no reason to believe that the three types of information manifestations, selective, structural, and taxonomic are the only possible or worth of attention. Whether the list will include other types of manifestation or not, every comprehensive study of information should be able to deal with all types.

The next topic for the study of information is its dynamics. When Shannon gave the name entropy to his measure of information on advice from Von Neumann based on formal analogy of the formulas, he was not aware of the fact that the foundations for the bridge between physics and information science have been already set in the analysis of "Maxwell's Demon." It was a long way to Landauer's assertion "Information is physical," but even those who insisted on separation of the disciplines had to accept the fact that Boltzmann's analysis of the Second Law of Thermodynamics has to apply to every information system implemented in physical reality. Thus, information has become a subject to temporal development leading to its destruction. It is a quite unusual situation, 
as in the past the fundamental measurable concepts have been entering scientific consideration typically through some conservation laws.

However, physical analysis of information systems and reversed informational analysis of physical phenomena is not the only direction of the study in information dynamics. Another direction started from Turing's study of computation. In both cases the fundamental concept is of a physical state. Any form of computation requires a system with multiple states, and typically it is understood that they are physical states. Thus, it is natural to expect that in information science the study of the relationship between the concept of a physical state and information is a necessary introductory step to the analysis of dynamics. With or without clarification of this issue, a general study of interaction between information systems can be expected as a main subject of information science. At present, there are multiple examples of such studies carried out within the limits of more specific disciplines, such as genetics, ecology, social sciences, economics, etc. The general approach is thus far limited to "kinematics" of information in the form of Maximum Entropy Principle, where development of one isolated system is considered. There is also considerable interest in open systems and systems far from equilibrium, but mathematical obstacles make the development of such theoretical systems slow.

The study of information dynamics requires a development of understanding of the relationship between information and causality. There was a considerable interest in the issues of causal relations involved in the analysis of information and its transmission or transformation. Another way to approach the relationship is to explain causality in terms of information.

\section{Examples of Philosophical Contributions to the Inquiry of Information}

Discussions of the problems in understanding the meaning of information are frequently influenced by the scientific mind-set of participants who believe that something which we call "information" exists independently from our inquiry in the real world and our role is just to discover what it is and to find its appropriate description independent from the descriptions of other objects of our experience. The active role of inquirer in creating possible multiple conceptualizations of information is usually excluded, and the discussion of the definition is limited to the question "who is right and who is wrong?" Thus, the interest is mainly in the literary understanding of the question "What is information?" without a reflection how this concept fits our understanding of all reality.

There are some exceptions in the study of information in which philosophical reflection is of special importance, although we may not be willing to follow the arguments of the authors. For instance, it is difficult to accept the view presented by Paul Young (1987) in the introduction and defended in the content to his book "The Nature of Information": "[...] in each and every case what we know as information is the precise equivalent of what traditionally has been referred to as form. [...] information must be viewed as a flow of mass-energy forms. [...] it will quickly become apparent that in all information processes in physical, chemical, and biological systems, the information stored, transmitted, or manipulated is identical with one or another of the above definitions of form - shape, configuration, pattern, arrangement, order, organization, or relations - so that whatever information is, it appears to be in all senses a form phenomenon." Young is trying to modernize Aristotelian concept of substance consisting of matter and form by identification of the former with the relativistic idea of mass-energy, and giving the latter dynamics of a flow. Not only this modern interpretation of Aristotelian substance is inconsistent, but there is no convincing argument that information and form should be identified. However, it is interesting to see that his understanding of form is focusing of structural relations, and is totally ignoring the original paradigm of Shannon's study of information involving the concept of probability.

Searching for the conceptual foundation for information in Aristotelian or scholastic concept of a form has been considered by others too. Raphael Capurro and Birger Hjorland started their inquiry in this direction from the etymological considerations. The word "information" has its origins in the idea that the substance acquire its individual, actual being contrasted with potentiality of the matter, through the process in which the latter is "informed." Scholastic philosophers viewed the process of learning as "informing the mind." 
The argument that the explanative power of this approach to information is supported by the fact of linguistic tradition is doubtful. The word "information" in this meaning disappeared from the language for several centuries. More persistent in the use has been the expression of "materialization of the form," than "information of the matter." Nobody not acquainted with scholastic philosophy wood be able to guess the meaning of the latter expression, and it is quite sure that when the word "information" has been revived by Shannon's work Aristotelian philosophy did not influence the way people understood it.

Thus, the etymology of the word "information" is irrelevant for our study, unless we have reason to believe that the revival of Aristotelian or scholastic philosophy can be helpful in building philosophical foundations for our present view of the world.

Since, I do not expect that much from the philosophy of the Peripatetics, my own view of information has followed different way. Let's try to look for what is common for all phenomena which are intuitively associated with information. The most fundamental common feature is the relationship between the multiplicity of potential options and the unique actualization.

This aspect of information is very clear in Shannon's study of communication in which each character of the message is carrying the amount of information dependent on the probability of its choice out of the multiplicity of an alphabet. Information here is identified with the selection of one out of many predefined options. Similar reference to the multiplicity can be found in the passage of Bateson's analysis of information, from which comes "any difference that makes a difference" frequently assumed to be his definition of information.

The relationship between one and many does not have to be limited to the selection of the one out of the many. We can also consider making one out of the many by introducing a structure which binds the many into a whole. Here we can find a correspondence with a form which gives individual existence to matter with its multiplicity of the potential forms of existence. Young in the quotation above "defines" form as one of many structures, each can be used to transform multiple components into a whole.

What we have found thus far is that whatever context of information is considered, information is related to the relationship between one and many, and the philosophical theme of the one-many relationship has probably the richest tradition of all philosophical themes. No philosophical system was indifferent to the questions relating unity and multiplicity. It is natural to expect that philosophical reflection on information conceptualized in terms of the one-many relation can be much more productive than that based on philosophical poverty of concepts such as uncertainty, difference, or even that based on as reach in philosophical reflection concept of the form.

Thus, author's own definition of information has been formulated as identification of a variety, where identification is understood as that which either selects, distinguishes one out of many, or that which makes the many into one (a whole). The former can be called a selective aspect of information, the latter structural aspect. (Schroeder, 2005) I have referred to the distinction as to an aspect of information, not a type, as the closer inspection shows that the "selectivity" and "structurality" of information are always present, and it is only a matter of manifestation of information which gives its dominating characteristic. Processes of transformation of information (processing) frequently are changing the dominating aspect.

We could see an example how the philosophical considerations can contribute to conceptualization of a subject which itself is of quite specific scientific interest.

\section{Between the Scientific and Philosophical Studies of Information}

Standard One of the most "philosophically loaded" domains of scientific inquiry of information is the study of consciousness. The concept of information has been for years a favorite tool in the attempts to identify and to understand the mechanisms responsible for conscious experience. Without doubts, the main reason for this choice has been the computer metaphor of the brain workings. Although in my own opinion the analogy between computation or computer processing and the work of the brain in the cognitive functions is deceiving, or even false, it is a clear fact that most of 
attempts to understand consciousness have been based on the assumption of some form of similarity in the architecture of the brain and computer. However, no matter whether the computer metaphor provides appropriate paradigm for the study of consciousness, there is no better way to investigate the subject, than to search for the mechanisms of information processing in the brain.

There is one characteristic of the conscious experience which from the early time of psychological research has been distinguished, its uniformity or wholeness. William James described it in short: "The perception is one state of mind or nothing." (James, 1896) Even earlier, the unity of cognitive processes has been expressed in the concept of apperception introduced by Gotfried $\mathrm{F}$. Leibniz, which in the $19^{\text {th }}$ Century J. F. Herbart has made a central subject of psychological study, and which emphasized the view that the mental experience is not composed of separate bits but forms a unity. One more step back takes us to Descartes and his center of common sense (sensus communis) integrating contributions of senses into a coherent perception, which of course has been inherited from Aristotelian study of the common sense and common sensibles. Actually, we can go back to the beginnings of European philosophical tradition to find the view similar to that of James presented by Xenophanes "If the divine exists, it is a living thing; if it is a living thing, it sees - for he sees as a whole, he thinks as a whole, he hears as a whole." (Barnes, 2001: 43)

In modern psychology, the question of the unity of consciousness diverged into many more specific fields of study such as a cross-modal sensory integration, the inherited from the Gestalt psychology question of the priority of the perception of a whole over the parts, the face recognition, etc.

It should not be a surprise, although it was very pleasant one for the author, that James in his search of the unity of consciousness has made quite extensive diachronic and cross-cultural study of the tradition of the one-many philosophy, which he identified with the opposition of pluralism and monism: "The alternative here [of the one and the many, $m j s$ ] is known as that between pluralism and monism. It is the most pregnant of all dilemmas of philosophy." (James, 1911/1948: 113)

It is a natural question to ask how this unity can be expressed in terms of information or information processing. The awareness of importance of the unity of consciousness has accompanied all modern development of psychology, but only quite recently first hypotheses of the possible models of information unity or integration have been presented.

Research on the unity of consciousness made by neuro-psychologists has not produced much material for theoretical considerations. Edelmann and Tononi (1998) with their collaborators have provided evidence of the correlations between firings of neurons and analyzed them in terms of entropy. However, the temporal correlations or identification of the regions of the brain cooperating in producing conscious experience do not tell us much about the mechanism which are responsible for the correlation. Also, the fact that the processes can be described in terms of entropy does not constitute evidence for any specific form of information processing. At best, we may be convinced that integration of information happens in the brain, but we do not have any clue how it happens.

Another approach, purely theoretical, was based on the assumption that cognitive processes must involve the only physical phenomenon clearly involving integration of the states of its components, the quantum-mechanical superposition. This approach culminated in the Hameroff-Penrose model of information integration in the brain. However, the years are passing and there is no convincing evidence that the relatively large regions of the brain responsible for cognitive functions could be considered quantum mechanical systems maintaining coherence for the time long enough to influence consciousness. (Tegmark, 2000)

Present author has proposed different approach which incorporates quantum-theoretical formalism into mathematical structure modeling information integration in the brain, but without necessity to assume that the brain or its parts are actually quantum-mechanical systems. (Schroeder, 2007\&2009) The approach is based on the assumption that important is not involvement of quantum mechanics as a physical theory describing the brain as a mechanical system, but the properties of the structure used in its formalism which can be used in the building of the model describing information integration. Since it is possible to identify a mathematical structure similar to that under- 
lying quantum formalism in the models of information processing, it is possible to transfer the concept of quantum coherence into the model of information processing. (Schroeder, 2006)

Search for explanation of the unity of consciousness and the attempts to describe or to model information integration in the brain are bringing a new theme into the study of information, its level of integration. The theme is of course of considerable interest for philosophical reflection.

At this point, it is too early to build any comprehensive system of thought based on the ideas of information integration, but the outlines of its description suggest a field for speculation. For instance, we can think about the distinction between essential properties and accidents (or distinction of a physical object and its physical states) in terms of the level of information integration. The essence (object) may consist of the information integrated into a whole, while accidents (states) would be the non-integrated part of information.

\section{Potential Contributions of Information Science to Philosophy}

There is another perspective in which the relationship between philosophy and information science can be considered. What kind of contributions can be expected from the latter to the former?

Certainly, the study of consciousness is a natural field of interaction between philosophy and science. For instance, the model of consciousness based on information integration can help in the elimination of the homunculus fallacy. (Schroeder, 2006) It is quite clear that this fallacy is a result of the assumption that the mechanisms responsible for cognition have the form of computer-like input-output devices, in which there is no essential difference between the information in-coming and out-coming. If we consider cognition as a process involving transformation of information into higher and higher level of integration, the process does not have any output. There is no need for the homunculus watching it.

Another classical source of trouble is the question about the free will. Here too, the model of consciousness based on information integration can help to eliminate the double paradox of the conscious subject being either fully, mechanically determined by its physical state, or equally frustrating possibility of being a completely random in its states. The solution would come with integration of information, which would make decision a result of integration of all information stored in the brain acting as a whole, in contrast to mechanical systems whose dynamics is determined by the current state and the forces of interaction with the environment.

Even more elusive, but still feasible within the range of current speculation based on the idea of information integration, would be the task to clarify the mind-body relationship. This could be achieved by making distinction between the physical state of the body identified with the volume of un-integrated information, and integrated information which would be identified with the conscious mind.

All examples above are speculative, as they are based on the assumption that we can find complete model of information integration with the identified functional elements of the brain on which the theoretical structure can be implemented. Thus far, models such as that proposed by the author are far from giving so specific picture. All what at present can be offered is a mathematical structure in which some elements can be identified with input channels of the low level of integration, and some other elements can be identified as carriers of highly or even completely integrated information. Moreover, it is unlikely, although not impossible, looking at the properties of the mathematical structure, that the elements of this structure could be identified with the functional units of the brain such as neurons. However, this issue requires more study, so hypothesizing about it is out of the scope of this paper.

\section{Conclusion}

The paper presented several theses regarding possible and actual developments in the process of solidification of the new scientific discipline of information science together with a review of methodological questions and a reflection on interactions between philosophy and scientific study of information. 
It seems that the most promising for acquiring a new perspective on the study of information are the interactions with the field of the study of consciousness, where the concept of information is applied to the analysis of mechanisms responsible for the conscious experience. In this context an interesting for both science and philosophy concept of information integration has been discussed here.

Information science needs firm foundations in a well defined concept of information. In this paper one of the possible ways to build such foundations has been proposed. Certainly, other alternative approaches should be explored.

\section{References}

Bar-Hillel, Y. \& Carnap, R. (1952/1963). An Outline of a Theory of Semantic Information. Technical Report No. 247, Research Laboratory of Electronics, MIT, 1952; reprinted in Bar-Hillel, Y. Language and Information: Selected essays on their theory and application. Reading: Addison-Wesley.

Barnes, J. (2001). Early Greek Philosophy. 2nd edition. London: Penguin Books.

Capurro, R. \& Hjorland, B. (2003). The Concept of Information. Annual Review of Information Science and Technology 37, $343-411$.

Frake, C. O. (1962). Cultural Ecology and Ethnography. American Anthropologist, 64, 53-59.

Hameroff, S. R. \& Penrose, R. (1996) Orchestrated reduction of quantum coherence in brain microtubules: a model for consciousness. Journal of Consciousness Studies, 3, 36-53.

James, W. (1896). The Principles of Psychology. Vol. 2. New York: Holt.

James, W. (1911/1948). The One and the Many. In W. James (Ed.), Some Problems of Philosophy. New York: Longman's Green and Co. Other parts of the study with the common title "The One and the Many" and appropriate subtitles, which appeared in several different publications of James can be found in: The Writings of William James: A Comprehensive Edition. J. J. McDermott (Ed.) New York: Random House, 1967, pp. 258-270.

Schroeder, M. J. (2004). An Alternative to Entropy in the Measurement of Information. Entropy, 6, $388-412$.

Schroeder, M. J. (2005) Philosophical Foundations for the Concept of Information: Selective and Structural Information. In Proceedings of the Third International Conference on the Foundations of Information Science, Paris 2005. Retrieved October 17, 2011 from http://www.mdpi.org/fis2005

Schroeder, M. J. (2006). Model of structural information based on the lattice of closed subsets. In Y. Kobayashi \& T. Adachi (Ed.) Proceedings of The Tenth Symposium on Algebra, Languages, and Computation (pp.32-47). Funabashi: Toho University. Significantly revised and enlarged version of this paper has been presented at the 14th Herbstakademie.

Wildbad K. (2007). Theory in Cognitive Neuroscience with the title: "Mathematical model of information integration in the brain based on the formal analogy with quantum coherence."

Schroeder, M. J. (2007). Logico-algebraic structures for information integration in the brain. Proceedings of RIMS 2007 Symposium on Algebra, Languages, and Computation (pp. 61-72). Kyoto: Kyoto University.

Schroeder, M. J. (2009). Quantum Coherence without Quantum Mechanics in Modeling the Unity of Consciousness. In P. Bruza et al. (Ed.), QI 2009, LNAI 5494 (pp. 97-112). Springer: Berlin.

Schroeder, M. J. (2010). Syllogistic Structure for Symbolic Representation of Information. 2nd International Congress on Square of Opposition, Corte, Corsica, June 17-20, 2010.

Shannon, C. E. \& Weaver, W. (1949/1998). The Mathematical Theory of Communication. Urbana: University of Illinois Press.

Tegmark, M. (2000). Importance of quantum decoherence in brain process. Physical Review, E61, 4194-4206.

Tononi, G. \& Edelman, G. M. (1998). Consciousness and Complexity. Science, 282, 1846-1851.

Young, P. (1987). The Nature of Information. New York: Praeger.

\section{About the Author}

Marcin J. Schroeder, Ph.D.

educated in Poland and United States in theoretical physics and mathematics. His research interests go beyond these original fields of expertise and include history and philosophy of science, culture studies, philosophical aesthetics, etc. In information science, his interests are focused recently on integration of information in all its aspects, from philosophical to mathematical modeling. Since 2004 Professor and Dean of Academic Affairs at Akita International University, Akita, Japan. 